\title{
Dynamic Design and Computer Imitation of Belt Conveyor with Horizontal Curves
}

\author{
Dan Wang ${ }^{12, \text { a }}$ Weigang Song ${ }^{1, b}$ Jie Liu $^{1, c}$ \\ ${ }^{1}$ School of Mechanical Engineering and Automation, Northeastern University, Shenyang 110004, \\ China \\ ${ }^{2}$ School of Mechanical Engineering, Shenyang University of Chemical Technology, \\ Shenyang110142, China \\ ªw1978@126.com, ’weigangsong@263.net, cjieliu@mail.neu.edu.cn
}

Keywords: belt conveyor with horizontal curves, tart-up process, braking process, dynamic design, computer imitation

\begin{abstract}
The dynamic design of a large belt conveyor with horizontal curves was studied in this paper. The method of calculating its running resistance was obtained by analyzing the orienting force and resistance on the curving parts of the belt conveyors. Dynamic equation of belt conveyor with horizontal curves by using discrete finite element model was established integrating the line-running parts and the curve-running parts. Additionally, dynamic analysis software of belt conveyor with horizontal curves was developed. Through simulation on real time system, start-up process and braking process were analyzed. The deviation of the belt was analyzed based on the tension, and the effect of curve running on belt conveyor was discussed.
\end{abstract}

\section{Introduction}

Because of the limitation of buildings or landform, the long distance belt conveyor may include horizontal curves. By setting idlers roller inclined forward, raising the inner curves, and increasing the groove angle, the natural orientation of the belt conveyors can be achieved. Up to now dynamic of long distance belt conveyor systems has been researched ${ }^{[1]}{ }^{[2]}{ }^{[3]}$, but the research on the dynamic of belt conveyors with horizontal curves is comparatively scarce. It gives the force analysis of the curve parts, deduces the calculating method of resistance, establishes the dynamic equations, develops the dynamic analysis software, simulates the real time system and then analyzes its results in this paper.

\section{The structure of the horizontal curves}

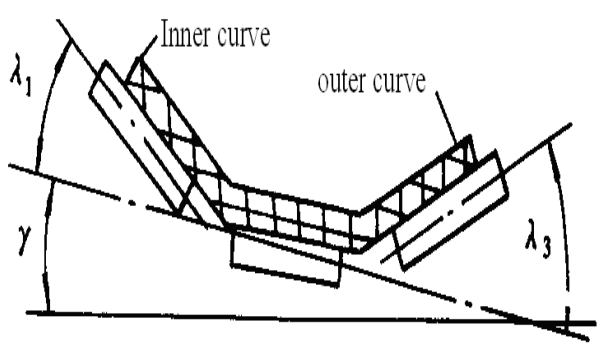

Fig. 1. The structure of superelevation angle in inner curve and trough angle of the idlers

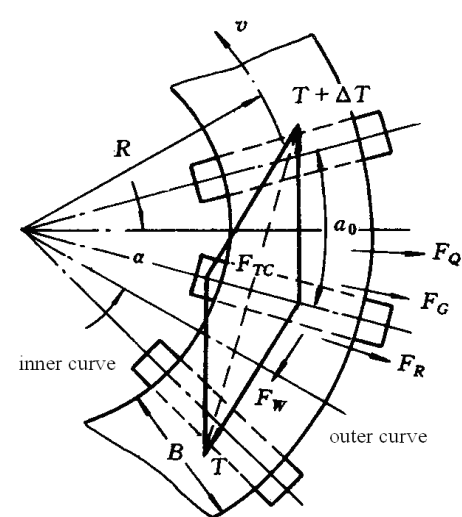

Fig. 2. Mechanical model of the belt at the horizontal curve 
The most effective centrifugal thrust force is produced by the gravity of belt and materials. The direction of the centrifugal thrust force is pointing to the outer curve; the groove angle of the idlers was increased as shown in fig.1. The inner of the idlers will be inclined along the moving direction of belt shown as fig2. If $F_{G}+F_{R}>F_{T C}$ is satisfied, the belt will not deviate to the inner curve.

\section{The running resistance calculation of the belt of the horizontal curves}

The running resistance includes the major resistance, the inclining resistance and the forwardinclining resistance of the idler. The resistance calculation process of the curve-running part can adopt the standard method ${ }^{[5]}$. Assuming the curve of the belt is circular arc, and supported by three idlers, as shown in fig.3.
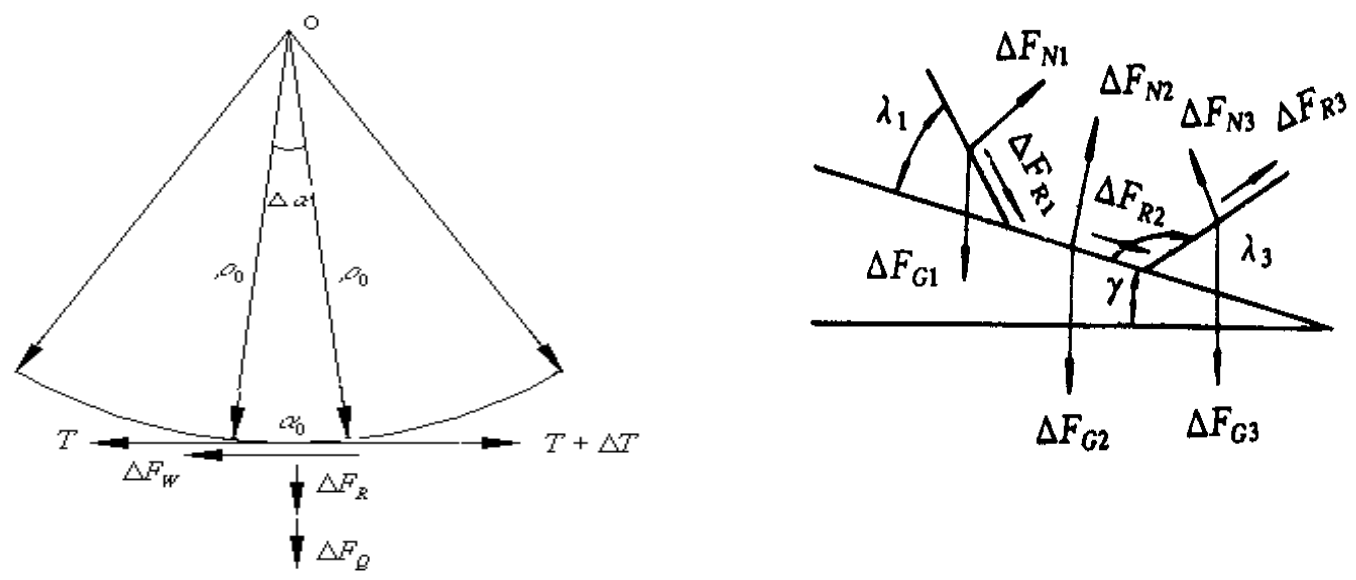

Fig. 3. Forced graph of the belt at the horizontal curve

The arc distance between two idler units is $a_{0}$, the radius of curvature is $\rho_{0}$, so the corresponding central angle is $\Delta \alpha=a_{0} / \rho_{0}$. The forces working on this period of belt include: belt tension $T, T+\Delta T$, running resistance $\Delta F_{W}$, gravity of the belt and material compressing on per idler $\Delta F_{G i},(i=1,2,3)$, support force of the per idler $\Delta F_{N i}(i=1,2,3)$, and the radial friction of the per idler $\Delta F_{R i},(i=1,2,3)$. Because the centrifugal inertial force is very small of the curves, it can be ignored. Aiming at the distance between adjacent idler units, according to the force analysis of fig.3

$$
\begin{aligned}
& \Delta F_{G i}=\Delta F_{N i} \cos \left(\lambda_{i}+\gamma\right)-\Delta F_{R i} \sin \left(\lambda_{i}+\gamma\right) \\
& \Delta F_{N i}=\frac{K_{i} q_{G B} g \rho_{0} \Delta \alpha}{\cos \left(\lambda_{i}+\gamma\right)-\mu_{3} \sin \left(\lambda_{i}+\gamma\right) \cos \varepsilon_{i}} \\
& \Delta F_{w}^{\prime}=f g\left[q_{R O}+q_{G B}\right] \rho_{0} \Delta \alpha \\
& \Delta F_{\varepsilon}=\mu_{3} \sum \Delta F_{N i} \sin \varepsilon_{i} \\
& \Delta F_{w}=\Delta F_{w}^{\prime}+\Delta F_{\varepsilon}=\left\{f g\left[q_{R O}+q_{G B}\right]+\mu_{3} \sum_{i=1}^{3} \frac{K_{i} q_{G B} g}{\cos \left(\lambda_{i}+\gamma\right)-\mu_{3} \sin \left(\lambda_{i}+\gamma\right) \cos \varepsilon_{i}} \sin \varepsilon_{i}\right\} \rho_{0} \Delta \alpha
\end{aligned}
$$

$\Delta F_{w}^{\prime}$ is the main resistance of loading section; $\Delta F_{\varepsilon}$ is the resistance because of idlers forward-inclining; $q_{G B}(\mathrm{~kg} / \mathrm{m})$ is the quality of belt and material per meter,; $K_{i}$ is partition coefficient of gravity $(i=1,2,3) ; \mu_{3}$ is the friction coefficient between idlers and belt; $\varepsilon_{i}$ is the forward-inclining angle of idlers $(i=1,2,3) ; \lambda_{i}$ is the groove angle of idlers $(i=1,2,3) ; f$ is the resistance coefficient of running. 


\section{Building up the dynamic equation of the belt conveyor with horizontal curves}

Belt conveyor with horizontal curves was divided in discrete finite units. The dynamic equation of the system is the integration of line-running parts, curve-running parts, drive unit and tension device. In this paper the emphasis is curve-running parts, and others can refer to reference ${ }^{[5]}$. Aim at the unit $i$ of curve-running parts. For this unit the $\operatorname{arc}$ is $l$, the radius is $R$, so the corresponding central angle is $\alpha=l / R$. The adjacent unit is unit $i-1$ and unit $i+1$. As shown in fig.4. So the dynamic equation of unit $i$ :

$$
m_{i} \ddot{u}_{i}+k_{i}\left(u_{i}-u_{i-1} \cos \frac{\alpha}{2}\right)+k_{i+1}\left(u_{i}-u_{i+1} \cos \frac{\alpha}{2}\right)+c_{i}\left(\dot{u}_{i}-\dot{u}_{i-1} \cos \frac{\alpha}{2}\right)+c_{i+1}\left(\dot{u}_{i}-\dot{u}_{i+1} \cos \frac{\alpha}{2}\right)+w_{i}=0
$$

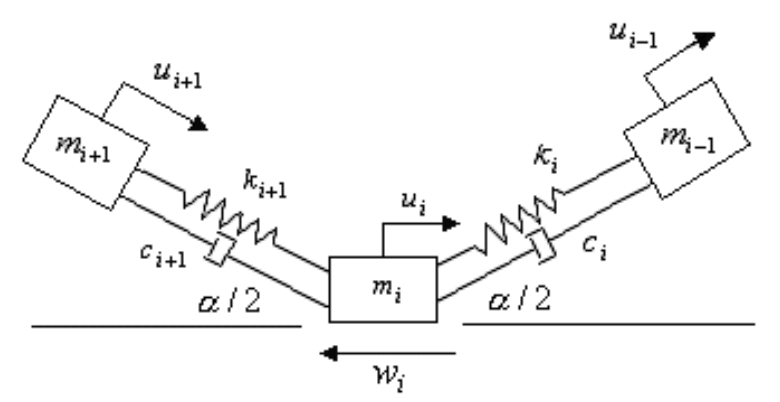

Fig. 4. Mechanical model of the unit $i$ in horizontal curves

$m_{i}$ is the quality of unit $i, w_{i}$ is its running resistance, $u_{i}$ is its displacement, $k_{i}$ and $c_{i}$ are the rigidity and damping between unit $i$ and unit $i-1$ respectively.

$$
w_{i}=\Delta F_{w i}=\Delta F_{w i}^{\prime}+\Delta F_{\varepsilon i}=\left\{f g\left[q_{R O}+q_{G B i}\right]+\mu_{3} \sum_{j=1}^{3} \frac{K_{j} q_{G B i} g}{\cos \left(\lambda_{j}+\gamma\right)-\mu_{3} \sin \left(\lambda_{j}+\gamma\right) \cos \varepsilon_{j}} \sin \varepsilon_{j}\right\} \rho_{0} \Delta \alpha
$$

Adding other parts of the system the dynamic equation of the whole system can conclude

$$
\mathbf{M u ̈}+\mathbf{C u}+\mathbf{K u}=\mathbf{F}
$$

\section{The simulation on real time system}

By the Wilson $\theta$ method the dynamic equation was solved. Using Visual Basic 6.0 the software was developed. Using the software the $8000 \mathrm{~m}$ belt conveyor with 2 horizontal curves was analyzed. The results are as follows:

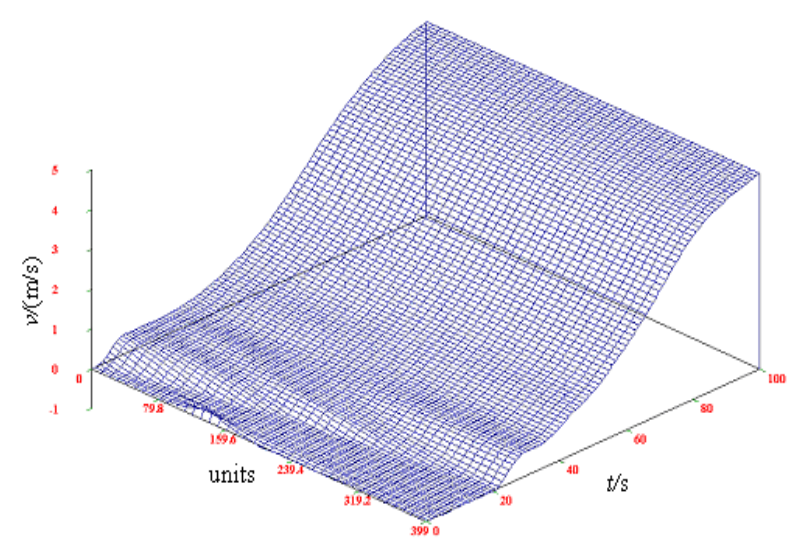

Fig. 5. Velocity curve of the belt of tart-up process

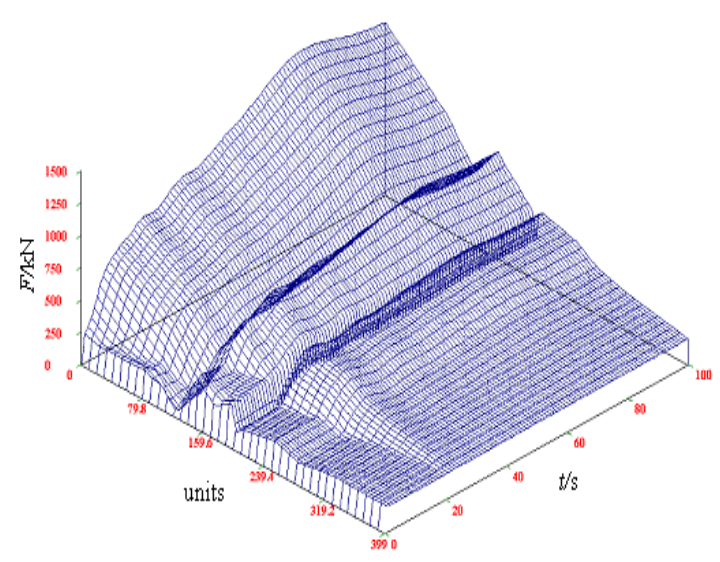

Fig. 6. Tension curve of the belt of tart -up process 


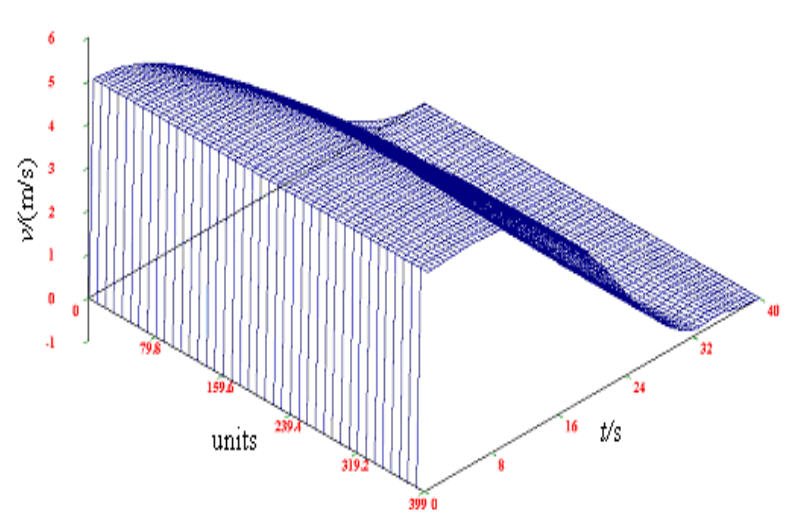

Fig. 7. Displacement curve of the belt of braking process

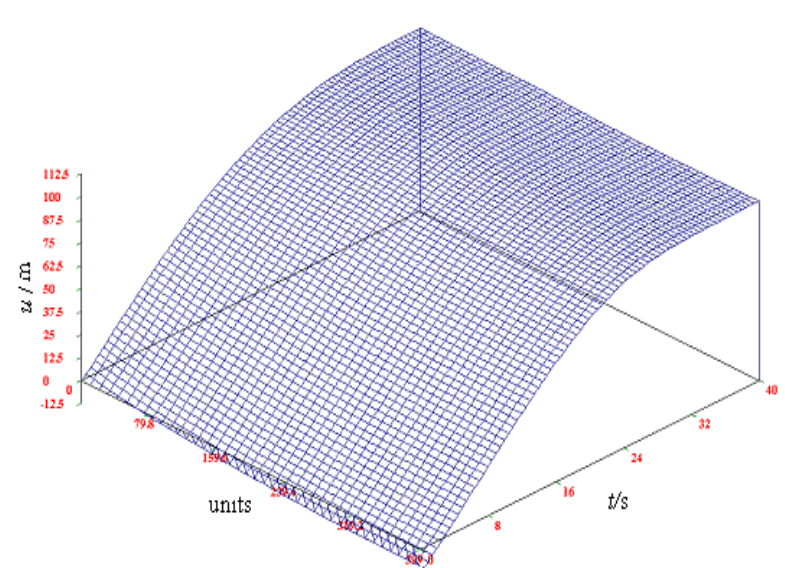

Fig. 8. Velocity curve of the belt of braking process

The comparison between line-running conveyor and curve- running conveyor was shown in table1. The tension of the belt was increased by the curve running, but the velocity and acceleration was not affected.

Tablel 1 The calculated result between line-running and curve-running conveyor

\begin{tabular}{lll}
\hline & line-running conveyor & curve-running conveyor \\
\hline $\begin{array}{l}\text { Maximum velocity of the curve's middle point } \\
/[\mathrm{m} / \mathrm{s}]\end{array}$ & 4.905 & 4.904 \\
$\begin{array}{l}\text { Maximum acceleration of the curve's middle } \\
\text { point } /\left[\mathrm{m} / \mathrm{s}^{2}\right]\end{array}$ & 0.103 & 0.104 \\
$\begin{array}{l}\text { Maximum tension of the curve's middle point } \\
/[\mathrm{kN}]\end{array}$ & 709.717 & 828.001 \\
\hline
\end{tabular}

According to the tension of start-up process the deviation of the belt was analyzed. Aiming at the middle point of the first curve, the tension of the fully loaded belt is:

$$
\begin{aligned}
& \Delta F_{N 1}=\frac{0.1801 \times 416.2 \times 9.8 \times 40.168}{\cos 40^{\circ}-0.2 \times \sin 40^{\circ} \cos 3^{\circ}}=46248.898 \mathrm{~N} \\
& \Delta F_{N 2}=\frac{0.6275 \times 416.2 \times 9.8 \times 40.168}{\cos 5^{\circ}-0.2 \times \sin 5^{\circ} \cos 2^{\circ}}=105012.113 \mathrm{~N} \\
& \Delta F_{N 3}=\frac{0.1924 \times 416.2 \times 9.8 \times 40.168}{\cos 40^{\circ}-0.2 \times \sin 40^{\circ} \cos 1^{\circ}}=32165.281 \mathrm{~N} \\
& \Delta F_{R 1}=\mu_{3} \Delta F_{N 1} \cos \varepsilon_{1}=0.2 \times 46248.898 \times \cos 3^{\circ}=9237.103 \mathrm{~N} \\
& \Delta F_{R 2}=\mu_{3} \Delta F_{N 2} \cos \varepsilon_{2}=0.2 \times 105012.113 \times \cos 2^{\circ}=20989.628 \mathrm{~N} \\
& \Delta F_{R 3}=\mu_{3} \Delta F_{N 3} \cos \varepsilon_{3}=0.2 \times 32165.281 \times \cos 1^{\circ}=6432.076 \mathrm{~N}
\end{aligned}
$$

Take the above values into following formula

$$
\begin{aligned}
& F_{R}=F_{R 2}+F_{R 1} \cos \lambda_{1}+F_{R 3} \cos \lambda_{3}=33825.068 \mathrm{~N} \\
& F_{G}+F_{R}=14279.216+33825.068=48104.284 \mathrm{~N} \\
& F_{T C}=11176.291 N
\end{aligned}
$$

So we can learn $F_{G}+F_{R}>F_{T C}$. Therefore, the fully loaded belt will not deviate to inner curve in the curve-running part. But it maybe deviates to the outer curve. In this case, vertical idlers will be adopted at outer curve. Then the belt conveyor will run normally. 


\section{Conclusion}

In this paper, the force on the curving parts of the belt conveyors was analyzed. Dynamic equation of belt conveyor with horizontal curves by using discrete finite element model was established. By simulating the actual system show that the software runs smoothly and the result is correct. The analysis results provide the basis for further research of the belt conveyor with horizontal curves.

\section{References}

[1] Harrison A, Roberts A W. Technical Requirements for Operating Conveyor Belts at High Speed [J],Bulk Solids Handling,1984,Vol.4(1) 232-234

[2] Nordell L K. The channer 20km overland-A Flagship of Modern Belt Conveyor Technology [J],Bulk Solids Handling,1991,Vol.11(4),342-347

[3] Zur T W. Viscoelastic Properties of Conveyor Belts - Modeling of Vibration Phenomena in Belt Conveyors During Starting and Stopping [J],Bulk Solids Handling,1986, Vol.6,№3 172-175

[4] Song wei-gang. Dynamic process research of large belt conveyor [J]. Journal of North-eastern University, 1996. (in Chinese)

[5] Song wei-gang. Design of belt conveyors transporting bulk material [M]. Shenyang: Publishing Company of North-eastern University, 2000. (in Chinese) 